\title{
La construcción del marco teórico y su relación con el enfoque metodológico de una investigación sobre la interacción social en la clase de Coordinación de Conjuntos Vocales e Instrumentales
}

María Angélica Bustos

\section{Introducción}

La búsqueda de logros y la preocupación por la eficacia de las estrategias didácticas en las investigaciones educativas pueden descuidar algunos aspectos como la definición del Marco Teórico y el establecimiento de relaciones con el Encuadre Metodológico-Investigativo.

No es necesario que el resultado de una investigación educativa sea la resolución de un problema didáctico, alcanzaría con que pueda llegar a ser una exhaustiva y metódica búsqueda que nos lleve a la comprensión del mismo. La construcción de relaciones entre lo teórico y lo práctico es una tarea que puede no brindar respues. tas inmediatas, pero brinda estructuras de pensamiento al investigador y educador musical que le sirven para resolver la práctica educativa. Comenzar por entender cuál es el conflicto es más que un comienzo.

Definir un Marco Teórico es asumir ciertas decisiones que están relacionadas con la postura pedagógica del docente investigador. La investigación-acción en el campo educativo presenta algunas dificultades metodológicas que exigen una clara correlación entre la postura ideológica como docente y como investigador. Si agregamos además que dentro de la educación nos enfocamos en lo musical, también habrá que ser coherente en decisiones que lo comprendan. Entonces ya tenemos tres disciplinas que hacer coincidir en la concreción de un trabajo de investigación sobre la enseñanza-aprendizaje de la música: la investigación, la pedagogía y la música.

Algunas decisiones referidas al método de investigación también dependen de la postura teórica asumida, como por ejemplo el tratamiento de los grupos de alumnos y la construcción de instrumentos para la recogida de datos que estos aportan. 
Este trabajo muestra las características del Marco Teórico de la investigación: La interacción docente-alumno como orientadora de aprendizajes en la clase de música. Un estudio de las interacciones en la clase de Coordinación de Conjuntos Vocales e Instrumentales $^{1}$; y describe relaciones establecidas con el Encuadre Metodológico, sus instrumentos y el tratamiento del grupo.

Se van a desarrollar ideas que cooperaron para el sostenimiento del Marco Teórico, estas pertenecen a los autores: Vygotski, Benveniste, Merleau Ponty y Grela, entre otros. No se van a presentar resultados, ya que ese no es el objetivo de este texto.

\section{Características generales de la investigación analizada}

La interacción docente-alumno como orientadora de aprendizajes en la clase de música es el estudio de las interacciones en la clase de Coordinación de Conjuntos Vocales e Instrumentales, recortando el siguiente caso: utilización de herramientas no verbales y verbales, y dentro de las últimas, de las manifestaciones del léxico musical, en el tratamiento de obras peruanas del barroco americano con el grupo de 1er año de la asignatura Coordinación de Conjuntos Vocales e Instrumentales en el Conservatorio de Música de la Ciudad de Pergamino.

Uno de los objetivos de la investigación fue el de estudiar las interacciones producidas mediante el empleo de herramientas verbales, como el léxico general y específico musical, y no verbales, como los instrumentos y la grafía musical, en el desarrollo de las clases de la asignatura Coordinación de Conjuntos Vocales e Instrumentales; observando qué aprendizajes musicales se manifiestan mediante el uso de cada tipo de herramienta.

Esta investigación se centra en la indagación de la interacción docente-alumno analizando particularmente el tipo y el contenido de las interacciones. Se ha escogido esta clase grupal porque en ella existe la posibilidad de observar no sólo las interacciones de los alumnos con el docente sino también las de los alumnos entre si; ya que el proceso se da en un contexto de trabajo colectivo con la necesaria conformación del grupo funcionando como tal. Uno de los objetivos fundamentales de la asignatura es el de enseñar a desempeñarse como parte de un conjunto en el acto de hacer música compartiendo un léxico específico. Para ello se emplean variadas herramientas, en el sentido vygotskiano. Estas herramientas cumplen una doble fun-

\footnotetext{
${ }^{1}$ Este trabajo de investigación corresponde a la Tesis presentada por María Angélica Bustos para completar la Maestría en Didáctica de la Música, en noviembre de 2009, en la universidad CAECE de Buenos Aires.
} 
ción porque se definen como herramientas en el proceso de enseñanza aprendizaje y también son herramientas para esta investigación. Algunas son verbales como la palabra y otras no verbales, como el código musical, o los objetos físicos como el cuerpo, los instrumentos musicales formales y no formales, los reproductores de música y la computadora.

La manipulación de estas herramientas posibilita diferentes tipos de interacciones con la consiguiente producción de variados textos: sonidos vocales e instrumentales, movimientos corporales más o menos codificados para interpretar parámetros del sonido, partituras y descripciones de las formas sonoras empleando un léxico específico.

La elección de obras musicales peruanas del barroco americano se debe a que el abordaje de este tipo de formas sonoras, por no ser de fácil acceso, favorece la apertura a nuevas interpretaciones semióticas, la búsqueda de otros textos, la cons. trucción de nuevas herramientas de análisis, y también porque para la comprensión de esas obras musicales se hacen necesarios la escucha, el análisis, la codificación en la reconstrucción de la partitura y la decodificación en la interpretación; recursos que se aplican a la elaboración de nuevos arreglos.

En esta investigación se propuso reflexionar sobre la diversidad de sentidos posibles de los variados textos generados por el uso de herramientas verbales y no verbales en la clase de música. En otros términos: la significatividad del aprendizaje musical puede estudiarse a través de una lectura semiótica de los textos que se generan en la interacción del docente con los alumnos. Esos textos, en un sentido amplio del término, pueden ser: consignas propuestas por el docente, ejemplos musicales de la propia ejecución o de otros intérpretes, textos literarios escogidos ad hoc, gestos, producciones de la ejecución musical de los alumnos, producciones verbales y grafías, entre otras manifestaciones.

Lo que motivó entonces la elección de este tema fue la búsqueda de una mirada diferente desde donde estudiar la interacción en la enseñanza-aprendizaje de la música, haciendo foco en el carácter instrumental de la interacción entre sujetos, en el sentido vygotskiano de los términos.

\section{El marco teórico}

El marco teórico se centra en conceptos pertenecientes a la psicología de Vygotski ampliados y complementados con los siguientes autores: Benveniste, Merleau Ponty, Watzlawick, Verón, Marc, abordando las siguientes temáticas: lingüística, fenomenología de la percepción, pragmática de la comunicación e interacción social. 
De Benveniste se toma como referencia el "El aparato formal de la Enunciación" interpretando que la lectura de un enunciado contempla una estructura formada por todos los elementos existentes en la escena. Estos son: el discurso o manifestación de la enunciación, la referencia que asocia a la lengua en la expresión de cierta relación con un objeto material o un hecho que pertenece al mundo, el locutor o enunciador que es el encargado de producir el enunciado, el acto mismo con su ubicación temporal-espacial y los instrumentos que la consuman, y por último, y no por ello se considera un orden valorativo en esta enumeración, está el alocutario, que es el destinatario de ese enunciado, que puede ser un otro, sujeto diferente al locutor, o bien éste se constituye en su propio alocutario en el monólogo. (Benveniste, 1985) Este aparato formal nos sirve de guía a la hora de pensar el acto de enseñar y aprender música como un acto de enunciación.

"Todo lenguaje se enseña, en definitiva, a sí mismo e importa su sentido en el espíritu del oyente. Una música o una pintura que a lo primero no se comprende acaba por crearse su público, si verdaderamente dice algo; es decir, por segregar ella misma su significación.” (Merleau Ponty, 1984, p 196) “...en la música no hay ningún vocabulario presupuesto, el sentido aparece vinculado a la presencia empírica de los sonidos." (Merleau Ponty, 1984, p 205) Estas citas de Merleau Ponty expresan la elección de una forma de mirar a la percepción musical junto con su lenguaje. Y esta mirada también incluye al léxico aplicado a la música porque diría Merleau Ponty que han escuchando la música que se oye quienes hayan dicho algo de lo oído, porque han constituido la realidad de la escucha construyendo sentido.

Watzlawick explicita que el estudio de la comunicación humana se puede organizar en tres áreas, establecidas por Morris, en: Sintáctica, que se centra en la transmisión información, los problemas de código, los canales, la capacidad, el ruido, la redundancia y otras propiedades estadísticas del lenguaje; Semántica que estudia el significado y la convención semántica, y por último la Pragmática, sosteniendo que toda comunicación afecta a la conducta. (Watzlawick, 1978, p 23)

Según la perspectiva psicosociológica la comprensión de la comunicación se ha enriquecido en muchas direcciones. Pudiendo ser la Comunicación total, verbal y no verbal, digital y analógica. Analizando la elaboración y reparto de significados, tomando al lenguaje como dimensión esencial de la cultura, siendo los valores, representaciones sociales y prácticas colectivas. Considerándola como el establecimiento de una forma de relación psicosocial entre dos personas, que considera la identidad social de los interactuantes. Y por último considerando el contexto que designa los hechos del lenguaje y los hechos sociales. (Marc y Picard, 1992 p 32, 33). 
Si bien todas estas ideas están presentes en las diferentes etapas de esta investigación, el pensamiento de Vygotski es su eje organizador. Porque estudiamos las maneras en que se manifiesta, en actividades musicales observables, la concepción semiótica de la conciencia y la relación aprendizaje-desarrollo dentro de la zona de desarrollo próximo según Vygotski. Para ello fue necesario definir en primer lugar los siguientes conceptos: herramienta y aprendizaje.

- Herramienta: La siguiente cita aporta un concepto básico sobre el carácter instrumental de la interacción entre sujetos: "Podríamos decir que las herramien. tas, los utensilios, son tan necesarios para la construcción de la conciencia como de cualquier artefacto humano. Permiten la regulación y transformación del medio externo, pero también la regulación de la propia conducta y de la conducta de los otros, a través de los signos, que son utensilios que median la relación del hombre con los demás y consigo mismo." (Riviere, 1988, p 41)

Para Vygotski "las herramientas semióticas más poderosas en el contacto social y la regulación interhumana de la conducta son las palabras. Son, en un sentido muy esencial, convenciones humanas construidas por la cultura para la comunicación e inter-regulación de la conducta" (Riviere, 1988, p 82) Aquí surge un interrogante: ¿y la música? ¿Qué tipo de herramienta es? ¿Es una forma de convención cultural con fines comunicativos? ¿Su manipulación incide sobre la conducta? Resulta necesario introducirse en este análisis para plantear la existencia de un carácter instrumental en sentido vygotskiano en la música, que se intenta describir en el siguiente recorrido.

La creación de la herramienta ha surgido del hombre para resolver los problemas que se le presentan. Le ha servido por ejemplo para adaptarse a un medio hostil o para modificarlo. Algunos sujetos se sienten motivados a crear una herramienta como respuesta, solución u opción para abordar la realidad, pero esa herramienta no es tal si no se sociabiliza, es decir, si no se comparte su uso con otros sujetos. El creador de la herramienta puede proponer una forma de uso y los otros sujetos pueden adoptar o transformar la manera de usarla o la herramienta misma. En ese proceso la herramienta es la mediadora en la interacción entre los sujetos. Imaginemos una situación concreta con un ejemplo factible en el campo musical. Podemos suponer que la herramienta es un objeto, un instrumento musical denominado "viola da gamba", que fue creado por un Luthier y otros sujetos instrumentistas abordaron su ejecución, y también otros Luthiers lo transformaron y lo llegaron a convertir en lo que hoy es el violonchelo. En este proceso el instrumento musical no ha sido la única herramienta que medió la interacción de esos sujetos, también se necesitó de la palabra, para que un Luthier pudiera decir a otro cómo hacer el 
instrumento nombrando sus partes u organizando la tarea de la construcción, y en ese proceso histórico de transformación de la viola da gamba llegar a modificar una o más partes lo suficiente hasta cambiar el nombre del instrumento por el de violonchelo. También el instrumentista utilizó la palabra para aprender y para enseñar a usar el instrumento porque debía nombrarlo, denominar sus partes y describir las características del sonido que producía o escuchaba para poder interactuar el discípulo con su maestro. No podemos suponer que las herramientas que usamos para abordar la realidad son solamente objetos materiales. La palabra es la herramienta fundamental que permite a los sujetos interactuar socialmente y actuar en la modificación de las herramientas materiales, así como de su uso. En el conocimiento musical se emplean herramientas verbales y no verbales, entre las verbales está el léxico específico, las no verbales son los objetos físicos como los instrumentos musicales y el código musical. Estas herramientas se aplican en un continuo devenir sensorial y cognitivo. En esta investigación se definen esos dos tipos de herramientas.

En la interacción docente-alumno en la clase de música el docente facilita al alumno diferentes tipos de herramientas para poder acceder a los conocimientos. Se pueden describir o enumerar algunas: instrumentos musicales cuya construcción y ejecución puede ser realizada por el alumno, herramientas de análisis para ser aplicadas en la escucha de una obra, realización de gestos para la dirección de la ejecución musical, lectura de textos orientadores, partituras para la ejecución, programas de escritura musical para PC, consignas direccionadas hacia la composición musical, entre otras, este listado no pretende ser exhaustivo sino ejemplificador de la variedad.

- Herramientas en esta investigación: Fundamentalmente se usan dos tipos de herramientas: verbales y no verbales.

Dentro de las herramientas no verbales, del tipo de los objetos físicos, empleadas en esta investigación se encuentran las fuentes sonoras: "cualquier tipo de dispositivo capaz de producir fenómenos sonoros"(Grela, 2006) Todo cuerpo vibrante, siempre y cuando la frecuencia y la intensidad de las ondas que producen estén dentro del rango audible es una fuente sonora. Las fuentes sonoras utilizadas aquí son: el cuerpo humano (el aparato fonador, las palmas, muslos y pies), los instrumentos musicales formales o no formales, y los aparatos eléctricos que producen o reproducen sonidos.

El cuerpo humano además de funcionar como una fuente sonora puede tener otra función como objeto físico, porque no sólo puede producir sonidos, sino que también 
puede representarlos. Las herramientas no verbales además de ser fuentes sonoras, o instrumentos como el cuerpo, pueden ser objetos que aunque no produzcan sonidos trabajan en relación con la tarea de la enseñanza aprendizaje musical.

Todas estas herramientas, del tipo de los objetos físicos, se focalizan en la sensorialidad tanto auditiva como visual y corporal. Con ellas se trabaja en el plano de la percepción.

Por otro lado están las herramientas que presentan un carácter de signo como lo son la codificación musical y las palabras, la primera es no verbal y la segunda verbal.

La codificación musical que se empleó corresponde a la escritura musical convencional: signos que representan los parámetros de altura, duración, timbre e intensidad de la música.

Con respecto a las herramientas verbales, se utilizó un vocabulario general sobre la música y un léxico específico que es el que corresponde al método de análisis del compositor Dante Grela.

El carácter individual de la sensorialidad hace que esta no se pueda compartir a menos que se transforme en palabra empleando un código común a otros sujetos. Las herramientas verbales permiten sociabilizar la percepción y se concretan en forma de léxico.

La manipulación de estos dos tipos de herramientas posibilita diferentes interacciones con la consiguiente producción de variados textos: sonidos vocales e instrumentales, gestos corporales más o menos codificados para interpretar los parámetros del sonido, partituras y descripciones de las formas sonoras empleando el léxico específico.

- Aprendizaje: Para Vygotski "el desarrollo de las funciones superiores exigiría (...) la apropiación e internalización de instrumentos y signos en un contexto de interacción. Y esto es aprendizaje." (Riviere, 1988, p 59) Así se evidencia una de las relaciones entre desarrollo y aprendizaje.

“La maduración, por sí sola, no sería capaz de producir las funciones psicológicas que implican el empleo de signos y símbolos, que son originariamente instrumentos de interacción, cuya apropiación exige, inevitablemente, el concurso y la presencia de los otros." (Riviere, 1988, p 59) En esta adquisición de signos y símbolos ese otro que aporta nuevos significados debe hacerlo dentro de la zona de desarrollo potencial, porque la exigencia de un aprendizaje inasible llevará al necesario replanteo de la tarea para poder modificar la interacción. Es decir, pretender que un niño de 4 años pueda hacer sonar un trombón cuando ni siquiera puede sostenerlo, o 
iniciarlo en la escritura musical cuando todavía no escribe su nombre, es no respetar la zona de desarrollo próximo. Estos son ejemplos extremos pero es muy común en la enseñanza de la música incurrir en errores de este tipo, tal vez no tan evidentes y por lo tanto más difíciles de descubrir.

El pensamiento y el habla están estrechamente ligados en una concepción semiótica de la conciencia, que postula la génesis, estructura y función del lenguaje interno a partir del habla. ¿Se podrán encontrar relaciones similares entre el pensamiento musical y su ejecución vocal o instrumental? ¿La interacción con el otro (sujeto) dentro de la zona de desarrollo próximo permite que pensamiento y ejecución musical se relacionen semióticamente en la conciencia? Se estudian estos interrogantes en el desarrollo de esta investigación.

Es importante que en este marco teórico se expliciten las ideas asumidas con respecto al rol docente, se considera no sólo pertinente sino necesario. En consecuencia se podría decir que el docente de música se debe mostrar como facilitador de herramientas, capaz de moverse dentro de la zona de desarrollo próximo al trabajar sobre el aprendizaje, y actuar como generador o motivador de la interacción instrumental es decir semiótica que se genera en el proceso de desarrollo-aprendizaje musical.

La zona de desarrollo potencial "no es otra cosa que la distancia entre el nivel actual de desarrollo determinado por la capacidad de resolver independientemente un problema, y el nivel de desarrollo potencial, determinado a través de la resolución de un problema bajo la guía de un adulto o en colaboración con otro compañero mas capaz" (Vygotski en Riviere, 1988, p 60) y "el buen aprendizaje es sólo aquel que precede al desarrollo. El concepto de 'zona de desarrollo potencial' sintetiza la concepción del desarrollo como apropiación e internalización de instrumentos proporcionados por agentes culturales de interacción." (Riviere, 1988, p 60)

- Relación Herramienta-Aprendizaje: Esta relación se manifiesta en la elección del Método de Análisis Musical de Dante Grela como herramienta de aprendizaje y se fundamenta en las características que a continuación se describen del mismo.

El análisis se constituye en una disciplina de fundamental importancia en relación con cualquiera de las diversas especialidades relativas a la teoría y a la práctica de la música. Esto se debe a que es la herramienta por excelencia que permite indagar sobre las características que hacen a la arquitectura de una obra musical, permitiendo así un abordaje de la misma en profundidad, ya sea desde un punto de vista práctico como lo es la ejecución musical o la pedagogía de la música, o bien desde una necesidad generada por las disciplinas teóricas de la profesión musical, tales como la musicología o la historia de la música. 
Por lo tanto, la posesión de una herramienta metodológica adecuada en dicho campo, resulta primordial dentro del proceso formativo del músico profesional, a fin de que la misma pueda cubrir adecuadamente las necesidades inherentes al examen analítico de formas sonoras con cualquier tipo de características.

Características, fundamentales a nuestro modo de ver en cuanto a su versatilidad como herramienta analítica de formas sonoras:

Posibilidad de aplicación para el examen analítico de formas sonoras pertenecientes a diversos períodos históricos, corrientes estéticas y compositores dentro de la música occidental, o a otras fuentes culturales tales como músicas étnicas, de Oriente, etc. Posibilidad de empleo amplia, en cuanto a las diferentes ramas de la teoría y la práctica musical". (Grela, D. 2006)

Luego de explicitar estos conceptos se llega a establecer como Eje de la Investigación al Léxico Musical, determinando como Unidad de Análisis a los Elementos léxicos referidos a la Forma Sonora tanto sean generales como específicos; para poder enunciar más tarde las Variables a analizar.

\section{El eje de la investigación-La unidad de análisis}

- Léxico General referido a la Forma Sonora: Este léxico se refiere al lenguaje musical básico que deviene de un manejo elemental de la lectoescritura musical, es de uso práctico para la lectura y escritura de la música en los primeros años del aprendizaje de la misma. Este léxico es con el que los alumnos habitualmente se manejan en la denominada Formación Básica, previa al ingreso al Profesorado. Un ejemplo es: melodía, notas, figuras, cuerdas, viento, percusión, carácter, altura, duración, parámetro/s, tónica, dominante, fuentes sonoras, compás/ases, ritmo, batido, 2/4, 4/4, cambio de compás, puntillo, semicorchea, corchea, clarinete, trompeta, modo, tonalidad, La Mayor, Re Mayor, la (nota), canto, intervalos, escala, grados conjuntos, nota, La menor, alteración, sonidos e instrumentos, entre otros.

- Léxico Específico referido a la Forma Sonora: Se considera léxico específico al aplicado cuando se trabaja sobre un tipo de música en particular o en el nivel de abordaje analítico aplicando el Método de Análisis de Grela.

Algunos de los términos léxicos son: arpa barroca, guitarra barroca, dulcian, bajoncillo, chab chas, sikus, quena, barítono atenorado, análisis paramétrico, viola da gamba, análisis articulatorio, análisis comparativo y forma sonora. 


\section{Las variables}

- Frecuencia: describe la cantidad de veces que aparece cada elemento léxico por clase.

- Modalidad de reemplazo: representa el reemplazo que utilizan los alumnos y la profesora cuando no emplean al elemento léxico correspondiente.

- Formas interactivas de recuperación / reformulación: del enunciado del docente en el enunciado del alumno; o del enunciado del alumno en el enunciado del docente.

\section{Análisis propuesto para las variables}

- Frecuencia: aparición en cada clase de los elementos léxicos generales y específicos, enunciados o reemplazados, por el docente y por los alumnos, previa intervención del docente o en forma espontánea. Se pretende verificar si en un análisis comparativo de los recuentos se advierte: disminución de intervenciones reformulativas del docente; y/o aumento del empleo espontáneo del los elementos léxicos específicos.

- Modalidad de reemplazo: es la forma en que se reemplaza el léxico, puede ser sintáctica o léxica.

- Sintáctica: reemplazo del elemento léxico adecuado por una formula, por ejemplo comparativa, que se acerque o se aleje del significado del término léxico específico.

- Léxica: si el reemplazo mantiene el paradigma (uso de un sinónimo); o si el reemplazo implica aproximación o alejamiento del concepto involucrado en el elemento léxico omitido; o el reemplazo es un gesto emblemático. "Los emblemas son aquellos actos no verbales que tienen una traducción verbal directa, o una definición de diccionario, compuesta habitualmente por una o dos palabras o quizás una frase. Esta definición o traducción verbal del emblema es conocida por todos los miembros de un grupo, una clase o una cultura." (Ekman, P. \& Friesen, W. en Verón y otros, 1971, p. 60).

- Formas interactivas de recuperación / reformulación: describen las situaciones de reformulación, estas son algunas de las posibilidades: a) El profesor interrumpe / corrige, el alumno reformula. b) El profesor no interrumpe / reformula luego modificando, el alumno repite o no. c) El alumno interrumpe su enunciado e introduce un gesto emblemático / el profesor introduce el termino léxico, el alumno reformula o repite. d) El docente no presenta el término léxico sino que formula características del mismo induciéndolo con preguntas, el alumno introduce el término léxico o no. 


\section{El encuadre metodológico}

Se aborda como estudio de caso el grupo de alumnos de 1 er año de la Asignatura Coordinación de Conjuntos Vocales e Instrumentales del Profesorado de Música para Nivel Inicial, Educación General y Polimodal del Conservatorio N ${ }^{\circ} 0001$ de la ciudad de Pergamino; en el lapso dedicado al desarrollo de la Unidad Didáctica Busquemos nexos entre la Música de América Latina del SXVIII y la de Europa, en el ciclo lectivo 2007.

Se realiza un estudio de las interacciones en las clases dadas en la primera parte de la Unidad Didáctica mencionada. Este recorte se fundamenta en la focalización del estudio de la adquisición de la terminología y el léxico básico para el análisis del repertorio abordado, ya que la segunda parte de la UD se desarrolla en función de la producción musical, producción de partituras, orquestaciones y arreglos, sobre la base del análisis del repertorio realizado en la primera parte, y requeriría de otro tipo de estudio.

Para abordar este trabajo se toman elementos tanto del estudio de las interacciones lingüísticas, como de las no verbales, considerando de estas últimas los gestos emblemáticos en particular, por poseer una "traducción verbal directa" (Ekman \& Friesen en Verón y otros, 1971, p 60). En el análisis de las obras musicales, así como de los fragmentos ejecutados por los alumnos, se aplicó el método de análisis propuesto por el compositor Dante Grela.

Los instrumentos que se aplicaron a la descripción se construyeron de forma tal que permiten extraer significados desde diferentes perspectivas. A partir de la descripción de los textos encontrados se buscaron relaciones que evidencian la construcción de la interacción docente-alumno en la clase de Coordinación de Conjuntos Vocales e instrumentales I y los efectos de dicha interacción.

\section{Sujetos}

El curso completo conformado por los alumnos pertenecientes a la asignatura Coordinación de Conjuntos Vocales e instrumentales I de primer año del Profesorado de Música para Nivel Inicial, Educación General y Polimodal en el Conservatorio № 0001 de la ciudad de Pergamino, en el ciclo lectivo 2007.

El grupo, integrado por tres mujeres y dos varones, se conformó como tal en el transcurso de esta investigación, debido a que el trabajo de campo se realizó durante las primeras clases del año. 


\section{Procedimientos}

En ningún momento se pensó en crear una situación de laboratorio, por el contrario, se quiso estudiar el recorrido de un procedimiento habitual con una documentación más completa en donde la docente a cargo era la investigadora. Por ello es que se decidió trabajar en una situación real con alumnos propios para propiciar un proceso de auto evaluación de la acciones de enseñanza, como uno de los resultados de la investigación.

Se comienza diseñando para el trabajo de campo una unidad didáctica denominada Busquemos nexos entre la Música de América Latina del SXVIII y la de Europa.

La temática abordada en la unidad didáctica demandó la aplicación del análisis musical como el que se propone en el Método de Análisis del compositor Dante Grela, herramienta necesaria de ser aprehendida por los alumnos para facilitar el entendimiento del repertorio abordado. Las formas sonoras escogidas: Tonada en Contracanto, Cachua La despedida, Tonada El Congo, Cachua serranita El Quechenacho, y Tonada La lata; pertenecen a la denominada Música Peruana de la Dominación Ibérica, lo que comúnmente se menciona como "barroco americano", por la influencia de la música barroca europea así como de de la música aborigen de Latinoamérica. En muchos aspectos de su estructura sonora se pueden encontrar elementos de ambas músicas. Este repertorio no cuenta con partituras originales completas, los grupos que han realizado versiones de las mismas toman como punto de partida el Códice de Fray Gregorio Dezuola (1640-1709) y el Códice Trujillo del Perú o Códex Martínez Compañón (1782-85), y generalmente no se conocen publicaciones de sus partituras, sólo algunas tablaturas muy sintéticas de los Códices que suelen circular. De allí reside la necesidad de aplicar una herramienta de análisis que permita extraer de la versión grabada, la información necesaria para comprender sus características. En esta investigación se empleó la versión en disco compacto El Diamante que es la llevada a cabo por el Albalonga Ensamble dirigido por Aníbal E. Centrangolo, no se emplearon tablaturas porque la docente y los alumnos construyeron sus propias partituras a partir de la audición.

Algunas de las características de estas formas sonoras son: instrumentos de diferente origen geográfico, diferentes formas de ejecución, textos en distintas lenguas, formas variadas de colocación de la voz en el canto, entre otras. Esta diversidad, si bien no es el eje de esta investigación, aporta mucho material para que los alumnos requieran la aplicación de variadas herramientas durante el proceso de aprendizaje. 
En el proceso de enseñanza-aprendizaje el trabajo se alternaba siendo colectivo, grupal e individual según lo requería cada actividad. Se propusieron fundamentalmente actividades de análisis a través de la audición y ejecución vocal instrumental grupal o individual con lectura de partitura.

Hubo dos elementos que se puede decir que respondían a una decisión que no se podía modificar y estos eran: el repertorio y la construcción del sikus, porque formaban parte del programa de la asignatura y se constituyeron además en herramientas escogidas para observar su uso en la clase; pero los demás admitían su modificación según las necesidades de los alumnos en el devenir de los encuentros.

A través del canto, la audición, la ejecución de instrumentos y el movimiento corporal se trabajaba el proceso de adquisición y corrección del léxico musical.

Básicamente se plantea el uso de las herramientas no verbales de tipo material, para llegar al dominio de las verbales a través de la interacción.

\section{Herramientas empleadas en el tratamiento del grupo}

a. Herramientas no Verbales: objetos físicos, código musical.

- Fuentes sonoras. Este tipo de dispositivo cuenta con un cuerpo vibrante, que es el que produce el sonido, y un cuerpo resonante que lo amplifica para hacerlo audible. Tanto la frecuencia como la intensidad de las ondas que produce debe estar dentro del rango audible para que sea una fuente sonora. Cumplen con ese requisito los siguientes objetos empleados: El aparato fonador, las palmas, muslos y pies; los instrumentos musicales: sikus, guitarra criolla, piano, bombo; y el aparato reproductor de discos compactos.

- Cuerpo humano. El cuerpo humano, además de poder producir sonidos, funciona como una herramienta cuando interpreta codificando la información auditiva en una manifestación de movimiento. Puede interpretar las variaciones del discurso musical en sus diferentes parámetros a través de diversos gestos corporales involucrando parte o la totalidad del cuerpo. Muchos de esos gestos están codificados socialmente, se van a analizar solo los gestos de tipo emblemático porque son los que se vinculan directamente al léxico, otra de las herramientas empleadas. Estos están asociados a: las representaciones propias de músicas pertenecientes a danzas con coreografías fijas, los modos de acción de determinadas fuentes sonoras y las representaciones de los parámetros del sonido, como los gestos de manos y brazos empleados para la dirección musical, que sirven para interpretar indicaciones de compás (batido), de velocidad, de intensidad y de textura musical. 
- Objetos. Afinadores, atriles, pizarrón, marcadores y herramientas para construir el sikus.

- Código Musical. Escritura de la música en la grafía convencional de pentagrama, melodías y ritmos escritos con este código, partituras organizadas en sistemas de pentagramas para la lectura en conjunto del sikus, la guitarra y el bombo.

b. Herramientas Verbales

- Léxico General. Este léxico corresponde a la terminología básica musical, el conocimiento de los nombres de los instrumentos y sus clasificaciones, el nombre de las notas, tonalidades, figuras, indicaciones de tempo e intensidad, toda esta terminología corresponde a la trabajada en la Formación Básica de los alumnos en el Conservatorio antes de ingresar a la carrera de profesorado.

- Léxico Específico. Son tanto los términos empleados para nombrar elementos correspondientes a la música perteneciente al Barroco Americano, como los pertenecientes al Modelo de análisis de Dante Grela.

Se decidió emplear el léxico y la metodología de análisis propuesta por Dante Grela porque es una herramienta metodológica para la formación del músico pro. fesional que se puede aplicar a repertorio musical diverso, ya que permite indagar en los elementos de una obra musical, empleando un léxico que no está atado a características de tipo histórico-musicales, y por esto puede cubrir adecuadamente las necesidades inherentes al examen analítico de formas sonoras con cualquier tipo de características. Se adapta a las necesidades de las diferentes especialidades formativas porque se aplica a diferentes actividades musicales como la composición, la ejecución musical, la didáctica de la música, la musicología o la historia de la música. Según el objetivo en el análisis propuesto el método prevé su adaptación indicando cuáles elementos deben ser tratados en profundidad y cuáles en superficie. (Grela, 2006)

\section{Tratamiento del Grupo}

Para poder realizar el estudio de las interacciones en la clase de Coordinación de Conjuntos Vocales e Instrumentales, se filmaron los encuentros con los alum. nos. La duración de las clases era de 2 hs 30 minutos con un intervalo de quince minutos de descanso, que a veces se utilizaba para las devoluciones individuales de las evaluaciones.

En la planificación, la primera parte de la Unidad Didáctica Busquemos nexos entre la Música de América Latina del SXVIII y la de Europa, constaba de cuatro clases, pero luego del diagnóstico, que se debió prolongar por la incorporación 
de alumnos, se amplió a una más. Se concretaron así cinco encuentros, y todos se cerraron con una síntesis dialogada de los aspectos abordados, recogiendo las opiniones de los alumnos para ser tenidos en cuenta y modificar la planificación de ser necesario.

Resultaron así un total de ocho horas y treinta y tres minutos filmadas.

La siguiente es una síntesis de las actividades desarrolladas en los encuentros:

Presentación dialogada de los temas de la Unidad Didáctica. Audiciones de las obras con consignas generales y explicación del análisis que propone D. Grela en su metodología de análisis musical. Para ejercitar los diferentes tipos: "Análisis Paramétrico", "Análisis Articulatorio" y "Análisis Comparativo" se diseñaron las Fichas 1 y 2, como herramientas de ejercitación.

- Ficha 1: 1) alturas: melodía y armonía: sucesión y simultaneidad (modo y tonalidad), 2) duración incluyendo características del ritmo y compás; y 3) timbre (denominación y origen de los instrumentos).

Cuadro1: Ficha 1: Análisis Paramétrico

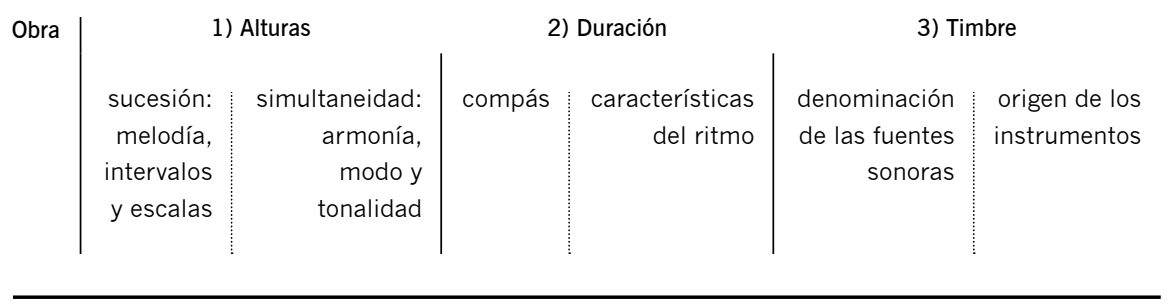

Cuadro 1: Ficha 1: Análisis Paramétrico. Mediante este tipo de análisis se describe la organización de un determinado parámetro en las formas sonoras.

Luego se amplía el análisis musical a la audición incorporando más elementos al análisis como se observa en la ficha 2:

- Ficha 2: 1) análisis articulatorio y comparativo; 2) análisis paramétrico: timbre, armonía e intensidad.

Cuadro 2: Ficha 2: Análisis Articulatorio, Comparativo y Paramétrico 
Cachua 1 1) Análisis articulatorio y comparativo: Mediante el análisis articulatorio

La se determinan las secciones y sub-secciones de la obra. Mediante el análisis

despedida comparativo se determina la denominación con letras de las secciones

track $N^{\circ} 1$ (mayúsculas) y sub-secciones (minúsculas) de la obra; teniendo en cuenta si estas son: iguales, semejantes o diferentes.

Tonada en

2) Análisis paramétrico: timbres (su distribución en la textura), armonía e intensidad. Mediante el análisis paramétrico se describe la organización de

contracanto

track $\mathrm{N}^{\circ} 2$ un determinado parámetro en las formas sonoras.

El diseño formal con la denominación de secciones y la descripción de cada parámetro dentro del mismo, se deberá realizar en hoja cuadriculada.

Cuadro 2: Ficha 2: Análisis Articulatorio, Comparativo y Paramétrico

Como ejemplo se les presenta un modelo de análisis con la indicación de compases en las secciones y la descripción de las características siguiendo las indi. caciones de la Ficha 2, para seguir a la audición el modelo de análisis y marcarlo en la partitura.

Construcción y afinación del sikus. Práctica del soplo.

Dictado a la audición de las obras. Escritura colectiva de las melodías.

Análisis musical a la audición en función a la trascripción a partitura de las obras, en clase y como trabajos prácticos para la ejercitación del dictado de melodías.

Lectura de lo trascripto con la ejecución comunitaria del sikus, ira y arca por separado.

Práctica de la ejecución con el Sikus y exploración de posibilidades en la textura con guitarra e instrumentos de percusión. Ejecución de "Tonada en contracanto": voz, sikus, guitarra y bombo con ejercitación de lectura a primera vista de la partitura, rotando los alumnos en la ejecución entre: siku ira, siku arca y guitarra, siendo el bombo ejecutado por la docente y reemplazando la parte de la voz por el sikus.

La evaluación de la ejecución se realizó empleando un Instrumento de evaluación de producto, elaborado "ad hoc", que contempla los siguientes Indicadores: 1) Adecuada calidad sonora (afinación, ataque y cuerpo del sonido, según el instrumento); 2) Precisión rítmica; 3) Precisión melódica (para los instrumentos de percusión se evalúa la interpretación de un ostinato con distribución de al menos dos registros: agudo y grave); 4) Correcta interpretación de las articulaciones y del fraseo; 5) Correcta interpretación de la intensidad y los planos sonoros; y 6) Ajuste en la coordinación grupal.

Con esta evaluación finaliza el trabajo de campo de la investigación. 


\section{Instrumentos utilizados}

Instrumentos y herramientas pueden cumplir una misma función, en esta investigación se habla de herramientas refiriéndose a las empleadas por los alumnos y la profesora durante los encuentros: herramientas verbales y no verbales; y se habla de instrumentos refiriéndose a los empleados para organizar y analizar la información recogida luego del trabajo de campo. Dicha información es la que se extrajo de las ocho horas y media de filmación.

La unidad de análisis está constituida por los elementos léxicos aplicados en la descripción de las formas sonoras. Son varios los instrumentos que se emplean, se construyen en función del estudio del léxico musical: su descripción, la frecuencia, el tipo de reemplazo, el modo de uso y la existencia de recuperación y reformula. ción del mismo.

Estos instrumentos diseñados para el análisis de las interacciones, dan cuenta del uso del léxico.

Se han elaborado tres matrices de datos en relación con la Unidad de Análisis y las Variables: Léxico General y Específico; Frecuencia; y Modalidad de reemplazo y Formas interactivas de Recuperación-Reformulación.

- Matriz: Léxico General y Específico: Esta matriz contiene todos los términos léxicos empleados en cada uno de los encuentros clasificándolos en generales y específicos. Es un cuadro de doble entrada, en la primera columna se indica el número de encuentro, en la segunda el léxico general y en la tercera el específico. Es una única matriz para todos los encuentros.

- Matriz: Frecuencia: La matriz Frecuencia da cuenta de la cantidad de veces en que aparece cada elemento léxico tanto sean los alumnos como la Profesora quienes los manifiesten. Es un cuadro de doble entrada indicándose en la primera columna las iniciales de los nombres de los sujetos que participan en cada encuentro y en la primera hilera conteniendo todo del léxico general y específico musical empleado. Muestra la cantidad de veces que aparece un término y en qué forma se manifiesta debido al color en que el número está escrito.

Se completan las casillas con números en color negro, verde, rojo y azul que representan cantidad de veces que aparece el léxico. En negro se indica la cantidad de veces que el léxico se enuncia verbalmente. En rojo se indica la cantidad de veces que el léxico no se enunció y se reemplazó en forma verbal tanto sea sintáctica o léxica. En verde se indica la cantidad de veces que el léxico no se enuncia y cuyo reemplazo es no verbal. En azul se indica la cantidad de veces que el léxico 
no se enuncia y se reemplazó gráficamente en la codificación de la lengua o en la codificación musical.

Se completó una matriz para cada encuentro, siendo cinco en total.

El siguiente cuadro es un fragmento de la matriz de uno de los encuentros, ya que la misma puede alcanzar a tener setenta y cinco términos léxicos empleados.

Cuadro 3: fragmento de la matriz frecuencia de uno de los encuentros.

\begin{tabular}{|c|c|c|c|c|c|c|c|c|c|}
\hline Léxico & & melodía con & & & & cuerpo & & & \\
\hline$\overline{\text { Sujeto }}$ & cuerdas & acompañamiento & viento & sikus & percusión & vibrante & parche & melodía & Sub total \\
\hline Prof & 3 & 1 & 2 & 7 & 3 & 1 & 1 & $1,6,5$ & $24+6$ \\
\hline Ev & 2 & 2 & 1 & & 1 & & 1 & 1,2 & $8+2$ \\
\hline M & & & & & & & & 3,2 & $3+2$ \\
\hline Ed & & & & 1 & & & & 2 & $1+2$ \\
\hline G & & & & & 1 & & 1 & 2 & $1+3$ \\
\hline Total & 5 & 3 & 3 & 8 & 5 & 1 & $2+1$ & $10+14$ & \\
\hline
\end{tabular}

Cuadro 3: Fragmento de la matriz frecuencia de uno de los encuentros

- Matriz: Modalidad de Reemplazo y Formas Interactivas de RecuperaciónReformulación: La segunda Matriz, Modalidad de reemplazo y Formas Interactivas de Recuperación-Reformulación, presenta dos variables: Modalidad de Reemplazo y Formas de Recuperación-Reformulación, que luego se analizarán por separado con toda la información recolectada. Se completó una matriz para cada encuentro, siendo cinco en total.

En ellas se exponen los léxicos enunciados o a los que se hace referencia; la modalidad de reemplazo del término léxico, si la hubiera, representada por el color en que se escribe el término mostrando específicamente cual fue el reemplazo empleado dentro de cada modalidad; el modo de empleo del término; y se explicita la recuperación y reformulación en los momentos en los que se observó en el proceso de enseñanza-aprendizaje. Esa recuperación o reformulación del léxico forma parte del producto de la interacción de los sujetos mediante el diálogo, ya sea del docente con los alumnos, como de los alumnos entre sí, es por ello que se indican los actores de la reformulación. 
En la primera columna del cuadro está la lista de sujetos que asistieron al encuentro, representados por sus iniciales. En la segunda columna se escribe el término léxico y según el color en que está escrito se puede saber si fue reemplazado o no y qué tipo de reemplazo se usó. En la tercera columna se describe el modo de empleo, que puede dar cuenta de si el alumno empleó espontáneamente el término, si lo repitió, si fue consecuencia de una pregunta enunciada por la profesora o si está dialogando con otro alumno. En la cuarta columna se ingresa el reemplazo, si lo hubiera, del término especificándolo. En la quinta columna se escribe la forma de recuperación y reformulación manifiesta mediante el diálogo, se indican los interlocutores y las frases textuales. Y en la sexta columna hay una referencia temporal que no se constituye en variable, sino que solamente tiene la finalidad práctica de facilitar la búsqueda en la grabación del momento en que ocurrió cada evento.

La tercera y la quinta columna presentan un contenido relacionado.

Las matrices se completan de la siguiente manera:

Solamente la segunda y la cuarta columna pueden completarse en colores. En negro se escribe el léxico que se enuncia verbalmente. En rojo, verde y azul se escriben los términos que no se enuncian y que han sido reemplazados. En color rojo se escribe el léxico cuyo reemplazo es verbal sintáctico o léxico. En color verde el léxico cuyo reemplazo es no verbal y la sustitución puede ser un gesto emblemático o una manifestación musical como el canto, el palmoteo, el tarareo o la ejecución de un instrumento. En azul se escribe el léxico que no se enuncia cuyo reemplazo es de tipo gráfico tanto en la codificación de la lengua como en la codificación musical, generalmente escrito en el pizarrón por la profesora o en los trabajos de escritura al dictado de los alumnos. Se indica en la columna correspondiente cómo se produjo la reformulación transcribiendo frases completas de los interlocutores que intervienen en la misma. Se separan con una doble línea cada proceso de reformulación-recuperación observado.

A continuación se transcribe un fragmento de la matriz que corresponde a un intervalo de tiempo en el que profesora y alumnos interactúan en proceso de reformulación:

Cuadro 4: Fragmento de la Matriz Modalidad de Reemplazo y Formas Interactivas de Recuperación-Reformulación de uno de los encuentros. 


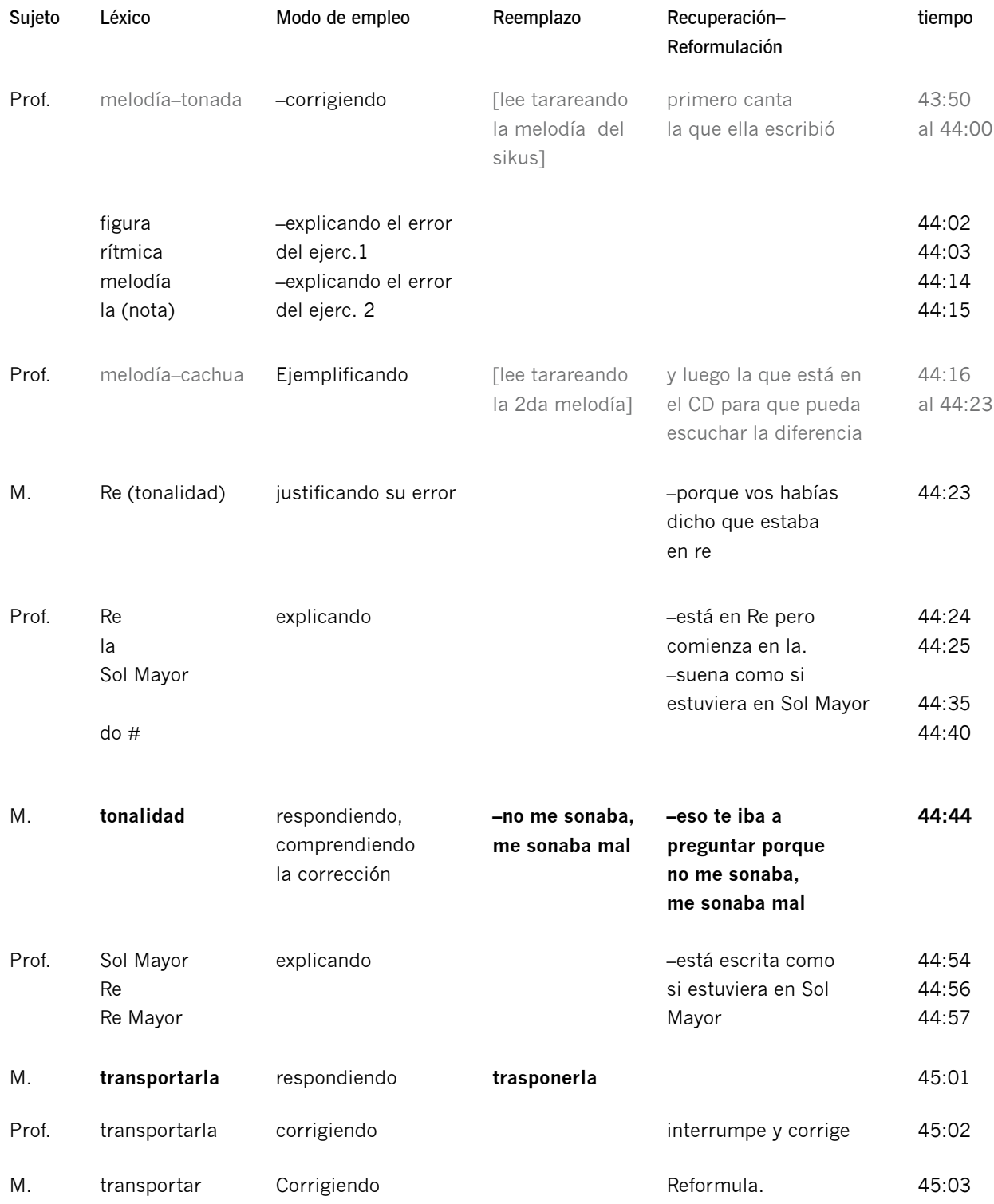

Cuadro 4: Fragmento de la Matriz Modalidad de Reemplazo y Formas Interactivas de Recuperación-Reformulación de uno de los encuentros 


\section{Reflexiones finales}

Luego de haber recorrido el encuadre Teórico y Metodológico, y el Tratamiento del grupo, tenemos una idea de las relaciones establecidas entre la teoría y la práctica investigativa del caso estudiado.

Se propone entonces reflexionar acerca de esas relaciones: ¿Qué elemento conecta a la forma escogida para el tratamiento del grupo de alumnos con las ideas de Vygotski? ¿Por qué los instrumentos para la recogida de datos nos pueden hablar de la postura de Merleau Ponty acerca de la escucha? ¿De qué manera se considera que las verbalizaciones del léxico musical son manifestaciones del pensamiento musical? ¿Qué lugar ocupa el Método de Análisis de Grela en el contexto teórico de la investigación?

A la primera pregunta sobre la relación entre las ideas de Vygotski y el tratamiento del grupo, podemos responder que la aplicación del concepto de herramienta es el que conecta los conceptos, ya que en todo momento la docente-investigadora fue consciente de las herramientas que escogía para el desarrollo de las clases.

A la segunda pregunta podemos responderla afirmando que los datos recogidos son términos pertenecientes a los enunciados que los alumnos y la profesora realizan sobre la música escuchada, la percepción se manifiesta en palabra cuando se hace sentido en ella.

Para la tercera pregunta se ha pensado que la demanda de transformar en palabra lo percibido completa un recorrido de pensamiento que con el solo hacer musical empírico no se alcanza. "La estructura del lenguaje no es el simple reflejo especular de la estructura del pensamiento. Por ello el pensamiento no puede usar el lenguaje como un traje a medida. El lenguaje no expresa el pensamiento puro. El pensamiento se reestructura y se modifica al transformarse el lenguaje. El pensamiento no se expresa en palabra, sino que se realiza en ella." (Vygotski, 1982:298)

A la última pregunta sobre el lugar que ocupa el Método de Análisis de Grela, se puede responder que es la herramienta que postula Vygotski y la palabra que demanda Merleau Ponty dentro del acto de enunciación que describe Benveniste. "La conciencia se refleja en la palabra lo mismo que el sol en una pequeña gota de agua. La palabra es a la conciencia lo que el microcosmos al macrocosmos, lo que la célula al organismo, lo que el átomo al universo. Es el microcosmos de la conciencia. La palabra significativa es el microcosmos de la conciencia humana." (Vygotski, 1982:347) Hacer consciente la escucha significa ponerle palabras a la misma, ese es el proceso del cual la investigación trató de dar cuenta, del proceso 
de aprendizaje musical que se realiza mediante la escucha y que se manifiesta con la verbalización de lo percibido, y para ello se tuvieron que hacer coincidir en este acto de enunciación a las ideas de los autores citados como herramientas del discurso que la investigadora escogió.

En conclusión ¿Dónde buscamos consejo para las decisiones metodológicas en la acción investigativa? Solemos pedirle al Marco Teórico que hable por nosotros y nos respalde en las determinaciones tomadas; si este es consistente y guarda una sólida relación con la Metodología, nuestra investigación se sostiene. Entonces sus resultados nos pueden guiar no solamente para resolver el problema que generó la investigación, sino también para tratar situaciones análogas.

El deseo de encontrar lo buscado no sólo puede guiarnos para encontrar las respuestas a nuestras preguntas, sino también puede llevarnos a encontrar respuestas a preguntas aún no formuladas.

El objetivo de este texto fue el de reflexionar acerca de las relaciones que la investigadora establece entre sus premisas teóricas y el tratamiento metodológico, generando así un espacio de discusión científica. Se espera que ustedes encuentren otras relaciones o también otros interrogantes y se los hagan llegar a la autora como devolución crítica de la lectura de este trabajo. 


\section{Bibliografía}

Benveniste E. (1999):

Problemas de Lingüística General I. Siglo veintiuno editores, SA de CV. 20a Edición.

Grela D.H. (2006):

Análisis Musical: Metodología y Práctica. Trabajo mimeográfico, facilitado por el autor para el "Taller de Audioperceptiva" dictado para la Maestría en Didáctica de la Música, marzo de 2006.

Merleau Ponty, M. (1984):

Fenomenología de la Percepción.

Barcelona: Planeta Agostini.

Marc, E. y Picard, D. (1992):

La interacción social. Buenos Aires:

Paidós.
Riviere A. (1988):

La psicología de Vygotski.

Visor Distribuciones, S.A. Madrid.

Watzlawick, P. y otros. (1978):

Teoría de la Comunicación Humana.

Ed. Tiempo Contemporáneo.

Verón, E. y otros. (1971):

Lenguaje y Comunicación Social.

Ed. Nueva Visión.

Vygotski L.S. (1982):

Obras Escogidas. Tomo II. Editorial

Pedagógica Moscú, Visor Distribuciones, S.A. 
María Angélica Bustos, "La construcción del marco teórico y su relación con el enfoque metodológico de una Investigación sobre la interacción social en la clase de Coordinación de Conjuntos Vocales e Instrumentales", revista del instituto superior de música 13 [ISSN 16667603]. Santa Fe, Argentina, Universidad Nacional del Litoral, marzo 2011, págs. 29-52. 